\title{
Síndroma de QT Longo: relato de caso de uma causa rara de síncope em idade pediátrica
}

Alicia Raquel Rebelo, ${ }^{1}$ Andreia Lopes, ${ }^{2}$ Catarina Magalhães, ${ }^{2}$ João Sarmento, ${ }^{3}$ Miguel Salgado ${ }^{2}$

\section{RESUMO}

Introdução: A síncope é um motivo frequente de preocupação em idade pediátrica. Apesar da sua etiologia ser maioritariamente benigna, é essencial excluir causas graves e potencialmente fatais que, embora raras, devem fazer parte do diagnóstico diferencial destas situações. Para isso, é necessário existir um elevado índice de suspeição.

Descrição de caso: Adolescente de 12 anos, previamente saudável, observado no serviço de urgência (SU) por primeiro episódio de perda de consciência (PC) com cinco minutos de duração e hipertonia generalizada. Mãe com episódios de PC classificada como epilepsia, sem outros antecedentes familiares relevantes, nomeadamente sem história familiar de patologia cardíaca em idade jovem ou morte súbita. Exame objetivo e eletrocardiograma (ECG) sem alterações, referenciado à consulta de neuropediatria onde realizou eletroencefalograma normal. Apresentou novo episódio de PC 18 meses depois, com recuperação espontânea em segundos, recorrendo ao SU. Repetiu ECG - ritmo sinusal e intervalo QT corrigido de 511 ms, tendo-se diagnosticado síndroma de QT Longo. Foi referenciado a consulta de cardiologia pediátrica e genética, tendo sido identificada mutação genética e medicado com beta-bloqueador. Após revisão da história clínica materna foi pedida avaliação por cardiologia, com confirmação do mesmo diagnóstico na mãe e na irmã.

Comentário: A síndroma de QT Longo caracteriza-se por um defeito da repolarização do miocárdio, associado a risco aumentado de morte súbita. As manifestações clínicas variam, desde ausência de sintomas até palpitações, síncope ou paragem cardíaca. Apesar da raridade, o diagnóstico é acessível através do ECG. No estudo de uma PC, a existência de um traçado de ECG prévio normal não invalida a sua repetição, uma vez que a alteração do intervalo QT pode ser inconstante. A existência de problemas cardíacos na família favorece a hipótese de síncope de causa cardíaca. Em caso de diagnóstico primário na criança deve ser efetuado um rastreio familiar de modo a despistar outros casos eventuais.

Palavras-chave: Adolescente; Síncope; QT Longo.

\section{INTRODUÇÃO}

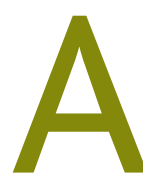

síncope define-se como uma perda de consciência abrupta e breve com resolução espontânea. Trata-se de uma condição frequente em idade pediátrica, estimando-se que até atingir a idade adulta $15 \%$ das crianças terão pelo menos um episódio de síncope. ${ }^{1}$ Esta manifestação clínica conta com múltiplas etiologias nesta faixa etária. Entre as causas mais frequentemente descritas encontram-se a síncope vaso-vagal, que corresponde a

1. Médica Interna de Pediatria. Hospital Senhora da Oliveira. Guimarães, Portugal. 2. Assistente Hospitalar de Pediatria. Hospital Senhora da Oliveira. Guimarães, Portugal.

3. Médico Interno de Cardiologia Pediátrica. Centro Hospitalar Universitário São João. Faculdade de Medicina da Universidade do Porto. Porto, Portugal. cerca de $50 \%$ dos casos, além da hipotensão ortostática ou a exposição a tóxicos. Raramente pode ser provocada por anafilaxia ou golpe de calor. Apesar de a maioria dos casos ser de etiologia benigna, as causas potencialmente fatais deverão ser obrigatoriamente excluídas. Habitualmente as causas graves de síncope são do foro cardiovascular, cursando com uma redução abrupta do débito cardíaco provocada por cardiopatia estrutural e/ou arritmia ou, menos frequentemente, bradicardia. ${ }^{1}$ Por sua vez, as taquiarritmias poderão estar relacionadas com fatores exógenos ou podem corresponder a anomalias eletrofisiológicas, como a síndroma de QT Longo. A síndroma de QT Longo tem uma prevalência estimada de 1 em cada 2.000 recém-nascidos saudáveis..$^{2-3}$ Neste contexto, e perante um doente 
em idade pediátrica que procura os cuidados de saúde por um episódio de síncope, a história clínica e exame objetivo minuciosos, complementados com eletrocardiograma, constituem passos essenciais na correta abordagem do doente, uma vez que, embora pouco frequentes, estas causas podem ser fatais.

\section{DESCRIÇÃO DO CASO}

Adolescente de 12 anos, previamente saudável, observado no serviço de urgência (SU) por primeiro episódio de perda de consciência (PC), em contexto de levante súbito, com cinco minutos de duração e hipertonia generalizada. Mãe com episódios de PC classificados como epilepsia. Sem outros antecedentes familiares relevantes, nomeadamente história familiar de surdez, patologia cardíaca em idade jovem ou morte súbita. O exame objetivo não demonstrou alterações e o eletrocardiograma (ECG) revelou-se normal para a idade. Foi referenciado à consulta de neuropediatria, onde realizou eletroencefalograma e cujo resultado não evidenciou alterações. Apresentou novo episódio de PC 18 meses depois, após se ter assustado com um toque de campainha. Recuperou espontaneamente em segundos, recorrendo novamente ao SU. Nesta altura apresentava exame objetivo sem alterações, repetiu ECG, que mostrou ritmo sinusal e intervalo QT corrigido prolongado (511 ms) (Figura 1). Foi referenciado a consulta de cardiologia pediátrica, onde realizou estudo de metabolismo fosfocálcico com doseamentos normais, holter de 24 horas e prova de esforço, tendo sido diagnosticada síndroma de QT Longo. Foi observado em consulta de genética, onde efetuou estudo molecular que identificou mutação em heterozigotia dos genes KCNH e ANK2, confirmando o diagnóstico clínico. Iniciou terapêutica farmacológica com nadolol e foi fornecida ao adolescente e à família a lista de fármacos desencadeadores de taquiarritmia, encontrando-se assintomático ao longo de 12 meses de seguimento. Após revisão da história clínica materna foi pedida avaliação por cardiologia, com confirmação do mesmo diagnóstico e identificação da mesma mutação genética. No rastreio de familiares diretos foi diagnosticada também a irmã com a mesma síndroma.

\section{COMENTÁRIO}

A síndroma de QT Longo é uma anomalia da repo-

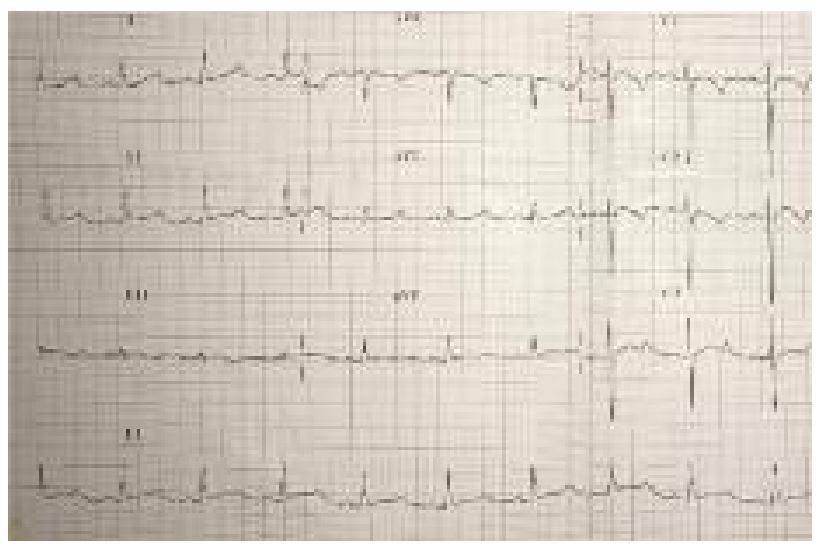

Figura 1. ECG do adolescente com prolongamento do intervalo QT.

larização miocárdica, podendo ser congénita ou adquirida. A sua forma congénita caracteriza-se por transmissão autossómica dominante (síndroma de Romano-Ward) ou recessiva (síndroma de Jervell e Lange-Nielsen), a primeira com atingimento unicamente cardíaco e a segunda associada a surdez neurossensorial, estando descritas várias formas da doença de acordo com a mutação genética identificada. ${ }^{4} \mathrm{O}$ diagnóstico é feito com base no prolongamento do intervalo QT determinado através de ECG, correspondendo a valores superiores a $460 \mathrm{~ms}$ na criança pré-pubere, $470 \mathrm{~ms}$ no rapaz púbere e $480 \mathrm{~ms}$ na rapariga púbere, associado a história clínica e antecedentes familiares que apoiam a suspeita clínica. ${ }^{5}$ Contudo, esta duração é influenciada por fatores como o estado autonómico, alterações elecrolíticas, fármacos e variações circadianas. Por este motivo, é possível a obtenção de um ECG sem alterações num determinado momento num doente com síndroma de QT Longo congénito. ${ }^{6}$ Embora possa ser assintomática, esta anomalia, por cursar com episódios de síncope e resultar em arritmia ventricular, confere aumento do risco de morte súbita. Classicamente associa-se a taquicardia ventricular polimórfica ou Torsade de Pointes, tipicamente desencadeadas por fatores ambientais como ruído, exercício ou stress. Estão descritos também fármacos que propiciam o seu desenvolvimento (Tabela 1), pelo que a educação do doente e familiares, alertando para este facto, é de extrema importância. ${ }^{4}$ Perante o diagnóstico de síndroma de QT Longo congénito deverá ser realizado rastreio aos familiares diretos, com realização de ECG e estudo 


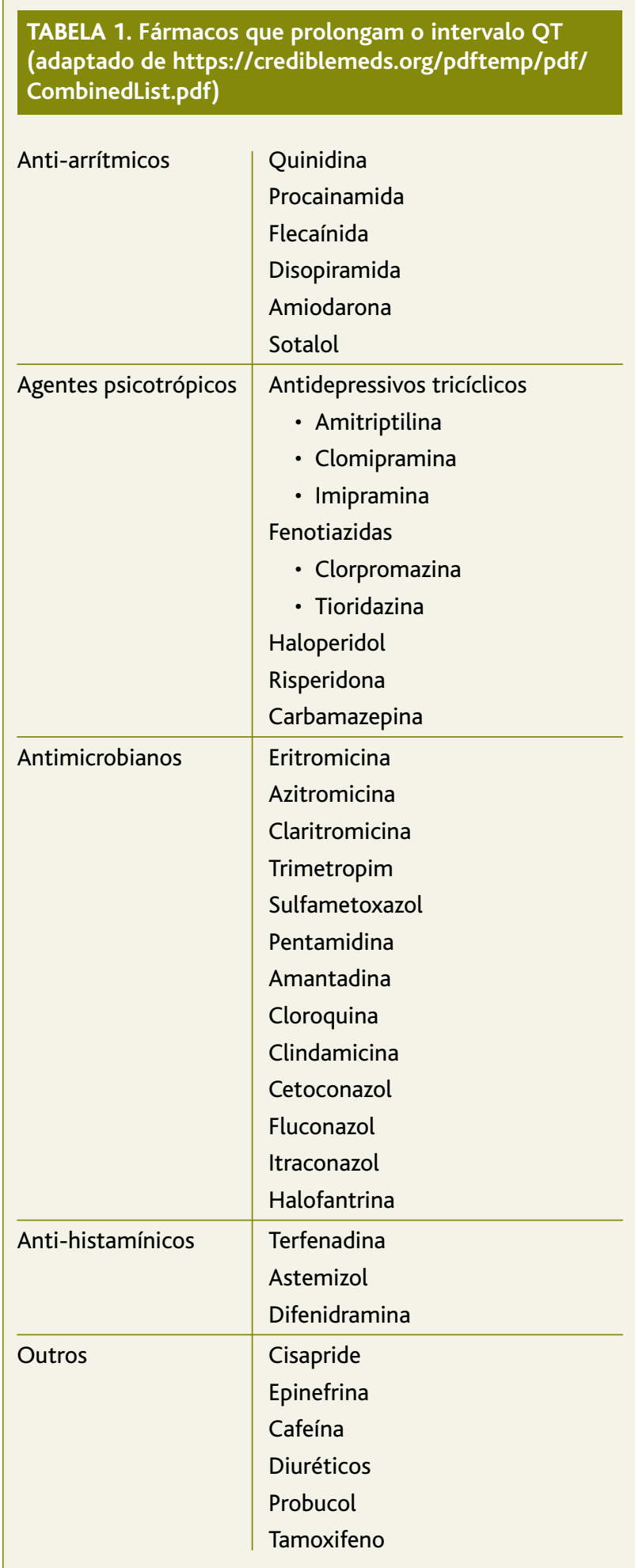

genético. A terapêutica passa pela administração e beta-bloquedores como nadolol, que mostrou ser mais eficaz comparativamente com outros fármacos desta classe. ${ }^{7-8} \mathrm{~A}$ dose habitual em idade pediátrica é de $1-1,5 \mathrm{mg} / \mathrm{Kg} /$ dia em dose única em doentes com 12 ou mais anos e dividida em duas tomas em doentes com menos de 12 anos de idade. ${ }^{9}$

O risco de eventos cardíacos não é completamente eliminado pela farmacoterapia. ${ }^{10}$ Nesse sentido, é fundamental promover modificações do estilo de vida, com evicção do exercício físico extenuante, reduzir exposição a sons altos abruptos (LQTS2) e evitar todos os fármacos que podem prolongar o intervalo QT. Geralmente os beta-bloqueadores são suficientes para controlar os eventos arrítmicos, mas pode ser necessário em casos raros realizar uma desenervação simpática esquerda ou implantação de CDI. O seguimento dos doentes deve ser continuado por toda a vida, com holter anual para despiste de eventos arritmicos e medição manual do QT. ${ }^{10}$

Embora sendo um relato de caso, esta descrição traduz vários dos problemas tipicamente associados a esta patologia, como a má interpretação da etiologia de episódios de síncope, a falha no diagnóstico tanto da mãe como da criança, além da importância do rastreio familiar (neste caso, com a mãe e irmã positivas), incluindo aconselhamento genético numa futura gravidez. Assim, os autores pretendem alertar para a doença que, embora rara, pode ser facilmente detetada com um simples ECG.

Em suma, perante um doente com episódio de síncope é fundamental excluir origem cardíaca, dado que, embora menos frequentes, estas etiologias podem ter um curso fatal.

\section{REFERÊNCIAS BIBLIOGRÁFICAS}

1. Salerno JC. Causes of syncope in children and adolescents. UpToDate [Internet]; 2019 Oct 8 [updated $2021 \mathrm{Mar}$. Available from: https:// www.uptodate.com/contents/causes-of-syncope-in-children-andadolescents

2. Schwartz PJ, Stramba-Badiale M, Crotti L, Pedrazzini M, Besana A, Bosi $\mathrm{G}$, et al. Prevalence of the congenital long-QT syndrome. Circulation. 2009;120(18):1761-7.

3. Schwartz PJ, Ackerman MJ, George Jr AL, Wilde AA. Impact of genetics on the clinical management of channelopathies. J Am Coll Cardiol. 2013;62(3):169-80.

4. Schwartz PJ, Ackerman MJ. Congenital long QT syndrome: epidemiology and clinical manifestations. UpToDate [Internet]; 2019 May 
23 [updated $2021 \mathrm{Mar}$. Available from: https://www.uptodate.com/ contents/congenital-long-qt-syndrome-epidemiology-and-clinicalmanifestations

5. Schwartz PJ, Crotti L, Insolia R. Long QT syndrome: from genetics to management. Circ Arrhythm Electrophysiol. 2012;5(4):868-77.

6. Ackerman MJ, Schwartz PJ. Congenital long QT syndrome: pathophysiology and genetics. UpToDate [Internet]; 2019 Apr 4 [updated 2021 Mar].Available from: https://www.uptodate.com/contents/congenitallong-qt-syndrome-pathophysiology-and-genetics

7. Schwartz PJ, Ackerman MJ. Congenital long QT syndrome: treatment. UpToDate [Internet]; 2019 [updated 2021 Jan 14]. Available from: https://www.uptodate.com/contents/congenital-long-qt-syndrometreatment

8. Chhabra L, Kowlgi NG, Kluger J. Beta-blocker variability in treatment of long QT syndrome. J Am Coll Cardiol. 2015;65(18):2053-4.

9. Ackerman MJ, Priori SG, Dubin AM, Kowey P, Linker NJ, Slotwiner D, et al. Beta-blocker therapy for long QT syndrome and catecholaminergic polymorphic ventricular tachycardia: are all beta-blockers equivalent? Heart Rhythm. 2017;14(1):e41-4.

10. Wallace E, Howard L, Liu M, O'Brien T, Ward D, Shen S, et al. Long QT syndrome: genetics and future perspective. Pediatr Cardiol. 2019;40(7):1419-30.

\section{CONFLITO DE INTERESSES}

Os autores declaram não possuir quaisquer conflitos de interesse.

\author{
ENDEREÇO PARA CORRESPONDÊNCIA \\ Alicia Raquel Rebelo \\ E-mail: aliciarebelo88@gmail.com \\ https://orcid.org/0000-0002-5713-2333
}

Recebido em 11-11-2019

Aceite para publicação em 12-08-2020

\section{ABSTRACT}

\section{LONG QT SYNDROME: A CASE REPORT OF A RARE CAUSE OF SYNCOPE IN PEDIATRICS}

Introduction: Syncope is a frequent motive of concern in pediatrics. Despite its mostly benign etiology, it is fundamental to exclude life-threatening causes.

Case report: We describe a 12-year-old boy brought to the emergency department because of a first syncopal event that lasted for five minutes with generalized hypertonia. His mother had a history of syncopal episodes who were classified as being epileptic, and there were no other relevant findings on family history. Physical exam and electrocardiogram (ECG) showed no anomalies. He was referred to a neuropediatric consult, where he underwent an electroencephalogram with a normal result. Eighteen months later he experienced another syncopal event that lasted a few seconds. He then repeated an ECG that showed sinus rhythm and a QTc of $511 \mathrm{~ms}$, leading to a diagnosis of long QT syndrome. He was referred to both pediatric cardiology and genetics consults, with identification of a genetic mutation, and was started on beta-blocker therapy. After studying his family, both his mother and his sister were confirmed as having the same diagnosis.

Comments: Long QT syndrome is defined as a disorder of myocardial repolarization, associated with an increased risk of sudden death. Children may range from being asymptomatic to experiencing palpitations, syncope, or even cardiac arrest. Despite its rarity, the diagnosis may be suspected on a regular ECG. The approach of a syncopal event in a child with a previous normal ECG does not exclude the diagnosis, as this electrocardiographic change can be intermittent. A positive family history supports the diagnosis. When the child is the first described case, his relatives should be evaluated.

Keywords: Adolescent; Syncope; Long QT syndrome. 